\title{
Änderungen der KLV und MiGeL per 1. Januar 2020
}

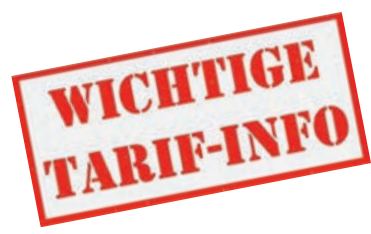

\section{Thomas Kessler}

FMH, Fachspezialist, Abteilung Ambulante Versorgung und Tarife

In diesem Artikel werden die wichtigsten Änderungen und Anpassungen der Krankenpflege-Leistungsverordnung KLV und der Mittel- und Gegenständeliste MiGeL per 1. Januar 2020 aufgezeigt.

Dieser Beitrag wurde am 7.2.2020 im Absatz «Änderungen der Krankenpflegeleistungsverordnung KLV per 1. Januar 2020» und dort im Unterpunkt «Krankenpflegeleistungs-Verordnung KLV" aktualisiert. Hier wurde ursprünglich aufgrund einer Meldung des Bundesamts für Gesundheit BAG von einer Änderung in Art. 12a Bst. c Ziff. 2 (Grippe-Impfung) berichtet, die jedoch durch ein späteres Korrigendum seitens BAG wieder rückgängig gemacht wurde (https://www.admin.ch/opc/de/official-compilation/2019/5017.pdf). Es liegt somit keine Änderung in Art. 12a Bst. c vor. Die entsprechende Passage wurde deshalb aus dem SÄZ-Beitrag entfernt.
Per 1. Januar 2020 traten Änderungen und Anpassungen der Krankenpflege-Leistungsverordnung KLV und der Mittel- und Gegenständeliste MiGeL in Kraft. Im nachfolgenden Text sind dabei die wichtigsten Änderungen und Anpassungen zusammengefasst. Weiterführende Informationen sowie Details dazu finden Sie direkt auf der Website des Bundesamtes für Gesundheit BAG.

\section{Änderungen der Krankenpflege-Leis- tungsverordnung KLV per 1. Januar 2020}

Krankenpflege-Leistungsverordnung KLV:

Für Art. 12e Bst. d KLV Früherkennung des Kolonkarzinoms sind neu auch die Früherkennungsprogramme der Kantone Basel-Stadt und Freiburg aufgeführt.

\section{Krankenpflege-Leistungsverordnung KLV Anhang 1:}

Weiterhin ist gemäss Punkt 1.3 Orthopädie, Traumatologie die Autologe Chondrozytentransplantation als Leistung in Evaluation für den Zeitraum 1. Januar 2020 bis 31. Dezember 2020 aufgeführt. Gemäss Punkt 2.1 Allgemein (Innere Medizin) ist die Extrakorporelle Photopherese nach einer Lungentransplantation nur bei Bronchiolitis-obliterans-Syndrom, wenn augmentierte Immunsuppression sowie ein Behandlungsver- such mit Makroliden erfolglos waren, eine Pflichtleistung in Evaluation für den Zeitraum 1. Januar 2020 bis 31. Dezember 2021. Gemäss Punkt 2.5 Krebsbehandlung ist die CAR-T-Zell-Therapie ab dem 1. Januar 2020 unter spezifischen Voraussetzungen eine Pflichtleistung. Gemäss Punkt 9.2 Andere bildgebende Verfahren (Radiologie) ist die Positron-Emissions-Tomographie (PET, PET/CT) bei der Fragestellung "Raumforderung" gemäss der klinischen Richtlinien der Schweizerischen Gesellschaft für Nuklearmedizin SGNM weiterhin vom 1. Januar 2020 bis 31. Dezember 2020 in Evaluation Zudem wird die Evaluationsphase Positron-EmissionsTomographie (PET, PET/CT) zur präoperativen Lokalisation von Nebenschilddrüsen-Adenomen bei primären Hyperparathyreoidismus nach negativer oder inkonklusiver konventioneller Bildgebung auf den 30. Juni 2020 verlängert. An der Liste der grundsätzlich ambulant durchzuführenden elektiven Eingriffe wurden einige Anpassungen gemacht, es verbleiben jedoch die sechs Eingriffsgruppen, welche per 1. Januar 2019 in Kraft gesetzt wurden. Details dazu können dem Anhang 1 der KLV unter Ziffer I entnommen werden.

Alle Änderungen der KLV inklusive Anhang 1 im Detail finden Sie unter diesem Link des Bundesamtes für Gesundheit BAG:

https://www.bag.admin.ch/bag/de/home.html Gesetze \& Bewilligungen / Gesetzgebung / Gesetzgebung Versicherungen / Gesetzgebung Krankenversicherung / Bundesgesetz über die Krankenversicherung / Änderungen in der Krankenpflege-Leistungsversicherung (KLV).

\section{Keine Änderungen der Analysenliste AL per 1. Januar 2020}

Auf den 1. Januar 2020 gibt es keine Änderungen der Analysenliste. 
FMH / Abteilung Ambulante Versorgung und Tarife Baslerstrasse 47

CH-4600 Olten

Tel. 0313591230 Fax 0313591238

tarife.ambulant[at]fmh.ch
Die Analysenliste im Detail finden Sie unter diesem Link des Bundesamtes für Gesundheit BAG: www.bag. admin.ch/al Themen / Versicherungen / Krankenversicherung / Leistungen und Tarife / Analysenliste (AL).

\section{Änderungen der Mittel- und Gegen- ständeliste MiGeL per 1. Januar 2020}

Im Punkt 2.3 Abgrenzung zu Leistungen anderer Sozialversicherungen wurde der folgende Text gelöscht: «Gemäss den Koordinationsregeln in den Artikeln 64 und 65 des Bundesgesetzes über den Allgemeinen Teil des Sozialversicherungsrechts (ATSG) sowie Artikel 110 KVV übernehmen die Sozialversicherungen jeweils Leistungen nach den Voraussetzungen des Einzelgesetzes. Sofern eine Leistung die Voraussetzungen von mehreren Gesetzen erfüllt, geht die Heilbehandlung im gesetzlichen Umfang und in nachste- hender Reihenfolge zu Lasten: 1. MV, 2. UV, 3. AHV/IV, 4. OKP.» Neu gilt: «In Bezug auf die Leistungskoordination der verschiedenen Sozialversicherungen wird auf Artikel 63 ff. des Bundesgesetzes über den Allgemeinen Teil des Sozialversicherungsrechts (ATSG) verwiesen.»

Gemäss Punkt 13 Hörhilfen gilt neu: Die Vergütung von Hörgeräten und den dazugehörigen Batterien erfolgt grundsätzlich gemäss den Bestimmungen (Vertragsbestimmungen, Tarif, Indikationsstufen) der AHV, IV oder UV (siehe auch Erläuterungen unter 2.3).

Alle Änderungen der Mittel- und Gegenständeliste im Detail finden Sie unter diesem Link des Bundesamtes für Gesundheit BAG: www.bag.admin.ch/migel Themen / Versicherungen / Krankenversicherung / Leistungen und Tarife / Mittel- und Gegenständeliste (MiGeL).

\begin{tabular}{|l}
\hline SAQM \\
ASQM
\end{tabular}

